\title{
Polarization-Dependent Chromatic Dispersion and Its Impact on Return-to-Zero Transmission Formats
}

\author{
Gino Biondini and William L. Kath
}

\begin{abstract}
We describe the extension of importance sampling and multicanonical Monte Carlo methods to events with large polarization-dependent chromatic dispersion (PCD), and we compare the efficiency of the two simulation techniques. We then use these methods to simulate pulse transmission in a return-to-zero system with full first-order polarization-mode dispersion compensation at center frequency. Results show that PCD does not contribute significantly to the statistics of the residual pulse broadening.
\end{abstract}

Index Terms-Importance sampling (IS), Monte Carlo simulation, optical fiber communications, polarization-mode dispersion (PMD), pulse broadening (PB).

\section{INTRODUCTION}

A MONG the techniques recently developed to increase the efficiency of Monte Carlo simulations of transmission penalties due to polarization-mode dispersion (PMD), importance sampling (IS) [1]-[4] and multicanonical Monte Carlo (MMC) sampling [5], [6] seem most promising. Neither of these methods, however, has so far specifically addressed polarization-dependent chromatic dispersion (PCD), which is known to produce transmission impairments. The issue is relevant since it is not clear what is the impact of large PCD events on system behavior. The purpose of this work is to address both of these questions.

After PMD compensation, SOPMD can become the main source of residual distortions [8]-[10]. Second-order PMD (SOPMD) is quantified by the frequency derivative $\tau_{\omega}$ of the PMD vector $\tau$ [7]. The component of SOPMD parallel to the PMD vector $\boldsymbol{\tau}_{\omega, \|}$ gives rise to PCD, while the perpendicular component $\tau_{\omega, \perp}$ results in depolarization. Since the depolarization is normally larger than PCD, efforts to maximize SOPMD typically result in large depolarization. Because PCD is also a source of impairments, however, comprehensive PMD tools should also be able to target large PCD. The growth of first- and second-order PMD is governed by the first two PMD concatenation equations [7], which for linearly birefringent elements are

$$
\begin{aligned}
& \boldsymbol{\tau}^{(n+1)}=R_{n+1}\left(\boldsymbol{\tau}^{(n)}+\Delta \tau_{n+1}\right) \\
& \boldsymbol{\tau}_{\omega}^{(n+1)}=R_{n+1}\left(\tau_{\omega}^{(n)}+\Delta \tau_{n+1} \times \tau^{(n)}\right) .
\end{aligned}
$$

Manuscript received February 14, 2005; revised May 13, 2005.

G. Biondini is with the Department of Mathematics, State University of New York, Buffalo, NY 14260-2900 USA (e-mail: biondini@buffalo.edu).

W. L. Kath is with the Department of Engineering Sciences and Applied Mathematics, Northwestern University, Evanston, IL 60208-3125 USA.

Digital Object Identifier 10.1109/LPT.2005.853016
Here, $\boldsymbol{\tau}^{(n)}$ and $\boldsymbol{\tau}_{\omega}^{(n)}$ are, respectively, the first- and second-order PMD vector after the $n$th section, while $\Delta \tau_{n+1}$ and $R_{n+1}$ are, respectively, the PMD vector and the Müller matrix of the $(n+1)$ th section. We consider a concatenation of $N$ such sections, with the length of each section taken to be Maxwellian-distributed; i.e., each component of $\boldsymbol{\Delta} \boldsymbol{\tau}_{n+1}$ is an independent identically distributed (i.i.d.) Gaussian random variable (RV) with zero mean and variance $\sigma^{2}=(\pi / 8 N)\langle\tau\rangle^{2}$, where $\langle\tau\rangle$ is the mean total differential group delay (DGD) of the line. This choice has been shown to result in a very efficient PMD generation model [3].

\section{IS AND MMC FOR PCD}

To apply IS, we first need to identify the most probable configurations that produce the events of interest, which in our case are large values of total PCD. As in [2]-[4], we employ a continuum limit of (1), (2), with $\mathbf{b}(z)=\lim _{z \rightarrow 0} \Delta \tau_{n+1} / \Delta z$ being the limiting value of DGD per section. The resulting equations can be solved exactly [3]. Unfortunately however, we are unable to find analytically the choice of $\mathbf{b}(z)$ that maximizes the total PCD. Let $\mathcal{U}=\left\{\mathbf{u}_{1}, \mathbf{u}_{2}, \mathbf{u}_{3}\right\}$ be the local reference frame such that $\mathbf{u}_{1}$ is aligned with $\boldsymbol{\tau}, \mathbf{u}_{2}$ with $\boldsymbol{\tau}_{\omega, \perp}$, and $\mathbf{u}_{3}$ with their cross product, as in [2] and [3]. A constrained maximum for the PCD exists if $\mathbf{b}(z)$ is restricted to be constant in both magnitude and direction [4]. Restricting oneself to constant biasing directions, however, is not an asymptotically efficient way to generate large PCD (nor large SOPMD). From (1) and (2), it is easily seen that nonzero PCDs are only generated when $\boldsymbol{\Delta} \boldsymbol{\tau}_{n+1}$ goes off the $\left(\mathbf{u}_{1}, \mathbf{u}_{3}\right)$ plane. (In contrast, DGD is maximized when $\mathbf{b}(z)$ is parallel to $\mathbf{u}_{1}$, and SOPMD when the orientation of $\mathbf{b}(z)$ varies linearly from $\mathbf{u}_{1}$ to $\mathbf{u}_{3}$ [1]-[4].) Parametrize $\mathbf{b}^{(n)}(z)$ as $b_{1}^{(n)}=b^{(n)} \cos \alpha^{(n)} \cos \beta^{(n)}$, $b_{2}^{(n)}=b^{(n)} \sin \alpha^{(n)}$, and $b_{3}^{(n)}=b^{(n)} \cos \alpha^{(n)} \sin \beta^{(n)}$. When $b^{(n)}=$ const, the choice of functions $\alpha^{(n)}$ and $\beta^{(n)}$ that yields the maximum PCD can be searched for numerically. As the number of sections increases, the maximum PCD is generated when $\beta^{(n)}$ varies linearly from 0 to $\pi$, and $\alpha^{(n)}$ is almost identically zero except for the last few sections. This suggests that the largest PCD is obtained by producing large SOPMD with almost zero total DGD, and then, at the last section, orienting $\boldsymbol{\Delta} \boldsymbol{\tau}_{n+1}$ parallel or antiparallel to $\tau_{\omega, \|}^{(n)}$ (for positive or negative PCDs, respectively).

The MMC method is based on the generation of random samples (here, $\boldsymbol{\Delta} \boldsymbol{\tau}_{n+1}$ ) via the Metropolis algorithm [11]. Let $X$ be the $3 \times N$ matrix containing the components of the individual PMD vectors of all birefringent sections. First, a trial configuration is generated by considering a random perturbation $X^{\prime}=X+\Delta X$. If we accept $X^{\prime}$ with probability $\min \left[1, p_{\{\Delta \tau\}}\left(X^{\prime}\right) / p_{\{\Delta \tau\}}(X)\right]$ and reject it otherwise, 

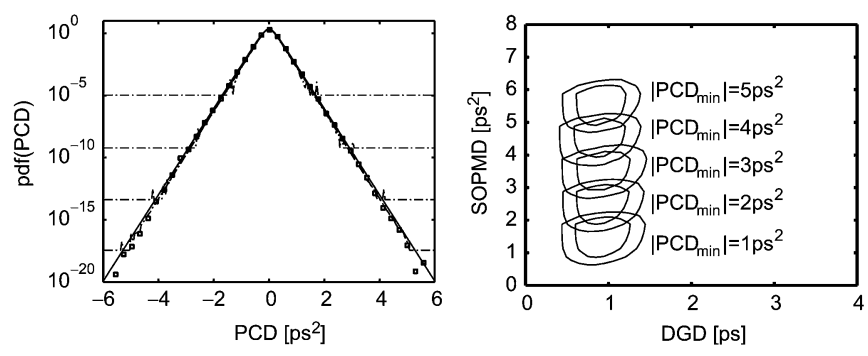

Fig. 1. Left: PDF of PCD as reconstructed via IS (squares) and MMC (four iterations, dashed lines) for a concatenation of 20 Maxwellian sections with total $\langle\tau\rangle=1 \mathrm{ps}$. Note the good agreement with the theoretical distribution [18] (solid lines) down to very low probabilities. Right: Regions of the (DGD, SOPMD) plane where the pdf of the PCD being in absolute value larger than $n \mathrm{ps}^{2}$ is larger than 0.8 and 0.6 of its peak value, for $n=1,2,3,4,5$, as reconstructed from importance-sampled Monte Carlo simulations.

where $p_{\{\Delta \tau\}}(X)$ is the probability density function (pdf) for $3 N$ i.i.d. zero-mean Gaussian RVs with variance $\sigma^{2}$, then $X$ follows a multivariate Gaussian distribution. MMC is a modification of the above procedure: After each iteration, (i.e., after a predetermined number of samples), probabilities are computed by sorting values into bins, and the resulting distributions are used to guide the next iteration [12]. In its simplest form, one modifies the acceptance probability as min $\left\{1,\left(p_{\{\Delta \tau\}}\left(X^{\prime}\right) / p_{\{\Delta \tau\}}(X)\right)\left(P_{\mathrm{pcd}}\left(\tau_{\omega, \|, \text { tot }}\right) / P_{\mathrm{pcd}}\left(\tau_{\omega, \|, \text { tot }}^{\prime}\right)\right)\right\}$, where $P_{\mathrm{pcd}}\left(\tau_{\omega, \|, \text { tot }}\right)$ and $P_{\mathrm{pcd}}\left(\tau_{\omega, \|, \text { tot }}^{\prime}\right)$ are the pdf of the total PCD as computed at the previous iteration, evaluated at the bins corresponding to $X$ and $X^{\prime}$, respectively. In each iteration, a new histogram is computed for each bin, and the probability associated with each bin is updated after the iteration is completed [12]. Note that Metropolis-generated samples are correlated; a sufficient number of steps must be used for the system to lose memory of the initial conditions (ICs). We also found it essential to discard an initial fraction of the configurations, to avoid a systematic bias of the pdf toward the ICs used for the algorithm.

Fig. 1 shows the pdf of PCD for 20 Maxwellian sections with $\langle\tau\rangle=1$ ps. For IS, in light of the above discussion, we used $\beta(z)=\beta_{\max } z / z_{\max }, \beta_{\max }=\pi, \alpha(z)= \pm(2 \pi / 5)\left(z / z_{\max }\right)^{\gamma}$, and $\gamma=5$, with $b=0$ (unbiased), 0.6, and 1.2 and 40000 samples for each case. For MMC, we took each element of $\Delta X$ to be a Gaussian RV with standard deviation $25 \%$ of $\sigma$; with this choice, roughly $30 \%$ of the perturbations were accepted. Four iterations were performed, each with 3000 steps (of which the first 1000 were discarded) for each of 1000 different ICs. For both IS and MMC, convergence is slower compared to the same methods applied to DGD and depolarization. The IS simulations, however, took $40 \mathrm{~s}$ on a single-processor workstation, while the MMC simulations required $8 \mathrm{~min}$ on the same machine.

\section{PUlse BroAdening After PMD COMPENSATION}

The temporal pulse broadening (PB) is defined as [13], [14] $B^{2}=\sigma_{\text {out }}^{2}-\sigma_{\mathrm{in}}^{2}$, where $\sigma^{2}=\overline{t^{2}}-(\bar{t})^{2}$ is the square of the root mean square (rms) pulsewidth, and the bar denotes the average of a quantity $Q$ with respect to the pulse intensity: $\bar{Q}=\int d t Q(t)\|\mathbf{u}(t)\|^{2} / \int d t\|\mathbf{u}(t)\|^{2}$, with $\mathbf{u}(t)$ being the pulse envelope's Jones vector. The PB is an often-used estimate of PMD-induced penalties [13]-[17], since it is usually well-correlated with penalties, but at the same time it is independent of the receiver. Neglecting input chirp, nonlinearity, and dispersion, the PMD-induced broadening is [14]

$$
B^{2}=\left(\overline{\tau^{2}}-(\mathbf{s} \cdot \bar{\tau})^{2}\right) / 4
$$

where $\tau$ is the input PMD vector, $\mathbf{s}$ is the pulse's input Stokes vector (which we take to be constant across the pulse), and here averages are performed with respect to the pulse's spectral intensity: $\bar{X}=\int d \omega X(\omega)\|\hat{\mathbf{u}}(\omega)\|^{2} / \int d \omega\|\hat{\mathbf{u}}(\omega)\|^{2}$, with $\hat{\mathbf{u}}(\omega)$ being the Fourier transform of $\hat{\mathbf{u}}(t)$.

When the mean DGD of the line is less than the pulsewidth, the PMD correlation bandwidth is larger than the pulse bandwidth, and $\tau$ can be approximately considered to be independent of frequency in (3). We refer to this as the "small PMD" limit. In this case, first-order PMD dominates, and $B=\sqrt{c(1-c)} \tau_{0}$, where $\tau_{0}$ is the DGD at center frequency and $c$ is the power splitting among the principal states of polarization. Then, because the DGD before compensation is Maxwellian and $c$ is uniformly distributed in $[0,1]$, the PB before compensation is Rayleigh-distributed [13]. Equation (3) also holds after compensation, but the DGD distribution after compensation is not Maxwellian. Thus, the pdf of the PB after compensation is not Rayleigh, even for small PMD. If variable-delay compensators are used to cancel the DGD at the center frequency, however, (3) can be expanded around the pulse's center frequency to show that, approximately (and assuming the pulse spectrum is symmetric), $B=(1 / 2) \omega_{\mathrm{rms}} \tau_{\omega}$, where $\omega_{\mathrm{rms}}^{2}=\overline{\Delta \omega^{2}}$ is the square of the rms pulse bandwidth and here $\tau_{\omega}$ is the magnitude of SOPMD at center frequency. Hence, the PB follows approximately the SOPMD distribution (a "sech-tanh" [18]). A similar relation is found for optimal first-order compensators, which use the frequency-averaged PMD vector [17]. These relations, however, are not valid for large PMD, i.e., when the PMD correlation bandwidth becomes smaller than the pulse bandwidth, which is when PMD is most likely to induce large transmission penalties.

\section{TRANSMisSion SimUlations}

To assess the impact of PCD on transmission penalties, we performed two sets of importance-sampled pulse transmission simulations. The first set ("without PCD biasing") used multiple biasings to obtain combinations of large DGD and/or SOPMD [4], with $b=0,0.5,1$, and $\beta_{\max }=0, \pi / 2$. The second set ("with PCD biasing") used the same technique with the addition of the biasing choice designed to obtain large PCDs, as described above. The input was a Gaussian-shape return-to-zero pulse with a full rms width of 5 ps (yielding an rms bandwidth of $200 \mathrm{GHz}$ ), and the mean DGD of the line $\langle\tau\rangle$ was varied between 1 ps ("small PMD") and 10 ps ("large PMD"). After transmission, the pulse was sent to a three degree-of-freedom (DOF) PMD compensator, with exact DGD cancellation at center frequency. A total of 180000 (10000) fiber realizations were used in the simulations with (without) PCD biasing, with 20 random input states of polarization per fiber realization. Fig. 2 shows the pdf of the residual PB after PMD compensation for small and large PMD, respectively; Fig. 3 shows, for the same cases, the joint pdf of PB and SOPMD, and Fig. 4 the joint pdf of PB and PCD, again after PMD compensation in both cases. For small PMD, Figs. 2(a) and 3(a) indeed show a good agreement betwen the numerical results and the relation $B=(1 / 2) \omega_{\mathrm{rms}} \tau_{\omega}$. As expected, for large PMD, the direct proportionality does not 

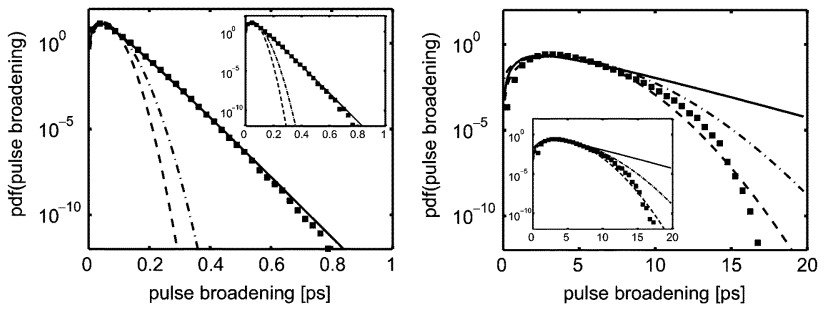

Fig. 2. PDF of PB after PMD compensation reconstructed from IS simulations (squares), for $\langle\tau\rangle=1 \mathrm{ps}$ (left) and for $10 \mathrm{ps}$ (right). In the first case, $\langle\tau\rangle$ is $20 \%$ of the pulsewidth, and the pdf agrees very well with the theoretical predictions. In the second case, $\langle\tau\rangle$ is twice the pulsewidth, and no theoretical predictions exist. Rayleigh (dotted-dashed lines), Maxwellian (dashed lines), and "sech-tanh" (solid lines) distributions with the same mean as the PB are shown. Insets: Simulations with just first- and second-order PMD biasing, i.e., without explicit PCD biasing.
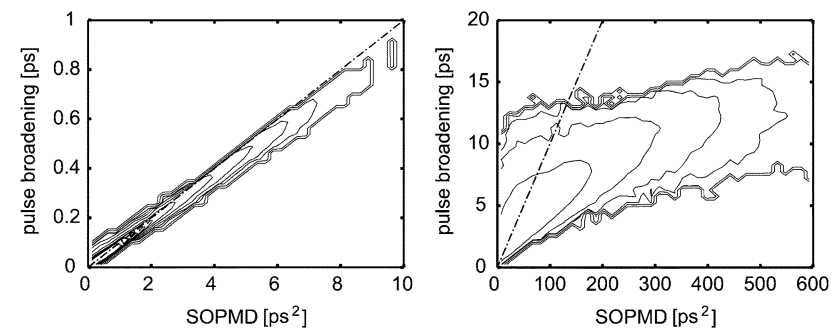

Fig. 3. Joint pdf of total SOPMD and residual PB after PMD compensation, for $\langle\tau\rangle=1 \mathrm{ps}$ (left) and for $10 \mathrm{ps}$ (right). Contour lines are at $10^{-n}$ with $n=$ $30,20,10,8,6,4,2,1,0.75,0.5,0.25,0.125,0$, and the maximum of the pdf is 46.2 (left) and 0.0077 (right). The dotted-dashed lines show the relation $B=$ $(1 / 2) \omega_{\mathrm{rms}} \tau_{\omega}$.
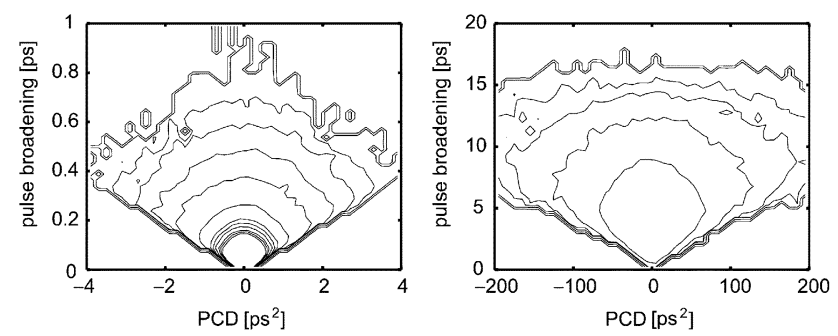

Fig. 4. Joint pdf of PCD and residual PB after PMD compensation, for $\langle\tau\rangle=1 \mathrm{ps}$ (left) and for $10 \mathrm{ps}$ (right). Contour lines are at $10^{-n}$ with $n=30,20,10,8,6,4,2,1,0.75,0.5,0.25,0.125,0$, and the maximum of the pdf is 27.1 (left) and 0.0062 (right).

apply, but a correlation still exists between PB and SOPMD. This should be compared to the lower correlation found between PB and PCD, even for small PMD. (In Fig. 4, regions of large $\mathrm{PB}$ exist for any value of PCD.)

Figs. 2-4 are obtained from simulations with PCD biasing. Simulations without PCD biasing, however, produce equivalent results for the PB for both small and large PMD (see insets of Fig. 2). This means that, even though explicitly biasing the PCD generates larger PCDs than does first- and second-order biasing, first- and second-order biasing is nonetheless enough to fully describe the transmission penalties in a system with up to three-DOF PMD compensation. (In contrast, biasing only the DGD is insufficient to generate full statistics for the penalties after PMD compensation [9].) This in turn suggests that, even though large PCD events do result in transmission penalties, their relative weight (compared to events with large DGD and/or SOPMD) is such that they are not significant in deter- mining the penalties' pdf. Note also that the depolarization is on average much larger than the $\mathrm{PCD}$, and if the $\mathrm{PB}$ is related to the total SOPMD, one can expect a stronger correlation with the depolarization than with the PCD. That this is indeed the case is confirmed by calculating the joint pdf of PB and depolarization, which results in plots almost identical to those in Fig. 3.

\section{CONCLUSION}

We extended IS and MMC to target large PCD events, and we performed numerical simulations of PMD-induced transmission effects in a system with PMD compensation, discussing the degree of correlation between PB, PCD, and SOPMD. Numerical results indicate that large PCD events are not significant in determining transmission penalties for PMD compensators with up to three DOF. Thus, when studying the performance of these devices, it is not necessary to explicitly bias for large PCDs if one is already employing first- and second-order PMD biasing. These results validate the multiple IS technique described in [2]-[4]. Nonetheless, expressely targeting large PCDs should be useful in special situations, for example, when testing the response of PMD compensators to specific types of distortions.

\section{REFERENCES}

[1] G. Biondini, W. L. Kath, and C. R. Menyuk, "Importance sampling for polarization-mode dispersion," IEEE Photon. Technol. Lett., vol. 14, no. 3, pp. 310-312, Mar. 2002.

[2] S. L. Fogal, G. Biondini, and W. L. Kath, "Multiple importance sampling for first- and second-order PMD," IEEE Photon. Technol. Lett., vol. 14, no. 9, pp. 1273-1275, Sep. 2002.

[3] G. Biondini and W. L. Kath, "Polarization-mode dispersion emulation with Maxwellian lengths and importance sampling," IEEE Photon. Technol. Lett., vol. 16, no. 3, pp. 789-791, Mar. 2004.

[4] G. Biondini, W. L. Kath, and C. R. Menyuk, "Importance sampling for polarization-mode dispersion: techniques and applications," J. Lightw. Technol., vol. 22, no. 4, pp. 1201-1215, Apr. 2004.

[5] D. Yevick, "Multicanonical communication system modeling-Application to PMD statistics," IEEE Photon. Technol. Lett., vol. 14, no. 11, pp. 1512-1514, Nov. 2002.

[6] - "The accuracy of multicanonical system models," IEEE Photon. Technol. Lett., vol. 15, no. 2, pp. 224-226, Feb. 2003.

[7] J. P. Gordon and H. Kogelnik, "PMD fundamentals: Polarization-mode dispersion in optical fibers," in Proc. Nat. Acad. Sci., vol. 97, 2000, pp. 4541-4550.

[8] W. Shieh, "On the second-order approximation of PMD," IEEE Photon. Technol. Lett., vol. 12, no. 3, pp. 290-292, Mar. 2000.

[9] A. O. Lima et al., "Statistical analysis of the performance of PMD compensators using multiple importance sampling," IEEE Photon. Technol. Lett., vol. 15, no. 12, pp. 1716-1718, Dec. 2003.

[10] E. Forestieri and G. Prati, "Exact analytical evaluation of second-order PMD impact on the outage probability for a compensated system," $J$. Lightw. Technol., vol. 22, no. 4, pp. 988-996, Apr. 2004.

[11] N. Metropolis et al., "Equation of state calculations by fast computing machines," J. Chem. Phys., vol. 21, no. 6, pp. 1087-1092, 1953.

[12] B. A. Berg and T. Neuhaus, "Multicanonical algorithms for first order phase transitions," Phys. Rev. Lett., vol. 68, no. 9, pp. 9-12, 1992.

[13] A. Galtarossa and L. Palmieri, "Relationship between pulse broadening due to PMD and DGD in long singlemode fibers," Electron. Lett., vol. 34, no. 492, 1998 .

[14] M. Karlsson, "Probability density functions of the DGD in optical fiber communication systems," Opt. Lett., vol. 23, no. 688, 1998.

[15] D. Mahgerefteh and C. R. Menyuk, "Effect of first-order PMD compensation on the statistics of pulse broadening in a fiber with randomly varying birefringence," IEEE Photon. Tech. Lett., vol. 11, no. 340, 1999.

[16] H. Sunnerud, M. Karlsson, and P. A. Andrekson, "Analytical theory of PMD compensation," IEEE Photon. Tech. Lett., vol. 12, no. 50, 2000.

[17] H. Sunnerud et al., "A comparison between different PMD compensation techniques," J. Lightwave Technol., vol. 20, no. 368, 2002.

[18] G. J. Foschini, L. E. Nelson, R. M. Jopson, and H. Kogelnik, "Probability densities of second-order PMD including PCD," IEEE Photon. Tech. Lett., vol. 12, no. 293, 2000. 